\title{
Ion-acoustic solitons in plasmas with two adiabatic constituents $\dagger$
}

\author{
FRANK VERHEEST ${ }^{1,2}$ and MANFRE A. HELLBER G \\ ${ }^{1}$ Sterrenkundig Observatorium, Universiteit Gent, Krijgslaan 281, B-9000 Gent, Belgium \\ (Frank.Verheest@UGent.be) \\ ${ }^{2}$ School of Physics, University of KwaZulu-Natal, Private Bag X54001, Durban 4000, \\ South Africa
}

(Received 30 October 2009 and accepted 16 November 2009, first published online 18 December 2009)

\begin{abstract}
Large amplitude ion-acoustic solitons are treated by a Sagdeev pseudopotential analysis, in a plasma with two adiabatic constituents, with the full inclusion of inertial and pressure effects for both. The sign of the supersonic species determines the polarity of the solitons, which are compressive in both constituents. Emphasis is placed on the determination of the soliton existence domains in compositional parameter space, allowing correct Sagdeev pseudopotential graphs to be easily generated, and offering insight into why limitations occur. Soliton velocities are bounded from below by the true acoustic velocity in the plasma model, and from above by the breakdown of the description when the supersonic ions reach their sonic point. Increases in the mass density ratio and the soliton velocity or decreases in the temperature ratio lead to increases in soliton amplitudes and decreases of the widths. Applications include hydrogen and electron-positron plasmas, and various kinds of dusty plasmas.
\end{abstract}

\section{Introduction}

Ion-acoustic solitons of large amplitude have been studied for several decades, using a pseudopotential description pioneered by Sagdeev and co-workers (Sagdeev 1966; Sagdeev and Galeev 1969). In this approach, the (positive) ions were described by the cold fluid continuity and momentum equations, whereas the electrons had Boltzmann distributions, i.e. neglecting ion pressure and electron inertia. Since one was looking for solitary structures that propagate unchanged, the description was given in a co-moving frame, in which the solitons appeared stationary.

With the help of the simplifying assumptions about the ion pressure and the electron inertia, it was possible to eliminate the fluid velocities between the stationary form of the continuity and momentum equations per species and express the densities in terms of the electrostatic potential. Substitution of the densities into Poisson's equation yielded, after one integration, the equivalent of an energy equation, involving the Sagdeev or pseudopotential (Sagdeev 1966; Sagdeev and Galeev 1969). The discussion of the latter was then given in terms of the classical

$\dagger$ It is a pleasure to dedicate this paper to Padma Shukla on the occasion of his 60 th birthday. 
mechanical analogy with the one-dimensional motion of a point particle in a conservative force field.

Over the years, various extensions of this formalism have been given, by investigating plasma models with additional or different constituents compared to those of standard proton-electron plasmas, by stressing the fluid-dynamical aspects of the nonlinear structures in an effort to better understand the physical limitations on their existence (McKenzie 2002; McKenzie and Doyle 2003; Verheest et al. 2004), by modeling more exotic configurations such as electron-positron (Pillay and Bharuthram 1992; Srinivas et al. 1996; Verheest et al. 1996) and dusty (Rao et al. 1990; Bliokh et al. 1995; Verheest 2000; Shukla and Mamun 2002) plasmas, and also, by incorporating ion pressure and/or electron inertia in the description.

The main stumbling block, specially if one wants to retain inertial and pressure effects for all species, is that the inversion of the momentum equations becomes analytically impossible, in the sense that one can no longer express the densities (or fluid velocities) as functions of the electrostatic potential, except for adiabatic pressure-density relations with polytropic index $\gamma=3$ (Mamun and Shukla 2002; Mamun 2008; Mamun et al. 2009; Tanjia and Mamun 2009).

Our main thrust in revisiting the description of large amplitude ion-acoustic solitons in a plasma with two constituents is to address the problem in a way which is physically transparent and places great emphasis on the determination of the existence domains in compositional parameter space, when both species are adiabatic, with the full inclusion of inertial and pressure effects for both. Once the existence regions are clarified, correct Sagdeev pseudopotential graphs can easily be generated, and one understands why they take the observed form, rather than having to rely on individual numerical tests, which offer no real insight into why limitations occur.

Our paper is structured as follows: in Section 2, we describe the model and introduce the relevant elements of the Sagdeev pseudopotential formalism. Section 3 is devoted to a thorough discussion of the physical limitations on the existence domains in compositional parameter space and of how variations in these parameters affect the soliton amplitudes and widths. Our conclusions are briefly summarized in Section 4.

\section{Model and Sagdeev pseudopotential formalism}

The nonlinear structure is moving with velocity $V$ in an inertial frame, where the undisturbed plasma is at rest, but for ease of computation we work in a co-moving frame, in which the nonlinear structure is stationary. We consider two adiabatic species, a supersonic (cooler) one and a subsonic (hotter) one, since we know that in order to have solitons, one needs plasma models having at least one supersonic and one subsonic constituent. It can easily be shown (Verheest et al. 2008) from the relevant fluid equations that in a frame where the nonlinear structure is stationary the density of adiabatic species is given by

$$
n_{s}=\frac{n_{s 0}}{2 c_{t s}}\left[\sqrt{\left(V+c_{t s}\right)^{2}-\frac{2 q_{s} \phi}{m_{s}}} \pm \sqrt{\left(V-c_{t s}\right)^{2}-\frac{2 q_{s} \phi}{m_{s}}}\right] .
$$

Here $n_{s}, q_{s}, m_{s}$ and $c_{t s}$ refer to the density, charge, mass and thermal velocity, respectively, of species $s$, with $n_{s 0}$ the undisturbed density far from the electrostatic 
soliton, and $\phi$ the electrostatic potential. We will use $s=c$ for the cooler supersonic ions and $s=h$ for the hotter subsonic species, respectively.

Expressions like (2.1) differ from the more commonly encountered ones in the literature, because we have followed (Verheest et al. 2008) the approach of Ghosh et al. (1996), as this leads to simpler and more transparent expressions for the densities. In (2.1) the \pm sign has to be interpreted as follows. The upper sign (here + ) has to be used for a subsonic species $\left(V<c_{t s}\right)$ and the lower sign (here -) for a supersonic one $\left(V>c_{t s}\right)$, in order that the correct limit, $n_{s 0}$, is obtained for $\phi=0$. This reasoning is based on the fact that $\sqrt{\left(V-c_{t s}\right)^{2}}=V-c_{t s}$ for $V>c_{t s}$ but becomes $c_{t s}-V$ when $V<c_{t s}$. In our model this implies that $c_{t c}<V<c_{t h}$ is needed (Verheest et al. 2004). Kinetic temperatures (expressed in energy units) will be introduced as $T_{s}=m_{s} c_{t s}^{2}$ and undisturbed adiabatic pressures as $p_{s 0}=n_{s 0} T_{s} / 3$ (McKenzie 2002; McKenzie and Doyle 2003; Verheest et al. 2004). We emphasize that the nomenclature $c$ (cooler) and $h$ (hotter) refers to the thermal speeds of the species, not to the temperatures (Verheest et al. 2007).

Examples could include electron-proton and electron-positron plasmas, for which $q_{c}=+e$ and $q_{h}=-e$, and various dusty plasmas where all electrons have been accreted onto supersonic, negative dust grains in the presence of positive ions or lighter dust grains, so that here $q_{c}<0$ and $q_{h}>0$. Parenthetically, we point out that we shall use $q_{h}<0$ and $q_{c}>0$ when plotting Sagdeev pseudopotentials in this paper. Interchanging the signs of the charges $q_{c}$ and $q_{h}$ leads to a change in the polarity of the nonlinear structures, but will not influence the existence conditions in the compositional parameter space, provided the normalization is suitably changed. In a plasma with two species, it is the sign of the supersonic species which determines the polarity of the solitons, regardless of the pressure closure hypothesis, as long as the pressure-density relation is polytropic (Verheest et al. 2004). Such solitons are always compressive in both constituents.

Charge neutrality in equilibrium means that $q_{c} n_{c 0}+q_{h} n_{h 0}=0$. We normalize the variables by introducing the dimensionless electrostatic potential $\varphi=-q_{h} \phi / T_{h}$, a 'Mach' number $M=V / V_{i a}$ in terms of a reference velocity (defined through $V_{i a}^{2}=$ $3 p_{h 0} / \rho_{c 0}=\left|q_{c}\right| T_{h} /\left|q_{h}\right| m_{c}$, where $\left.\rho_{s}=n_{s} m_{s}\right)$, a mass density ratio $\mu=\rho_{h 0} / \rho_{c 0}$ and a pressure or temperature ratio $\tau=p_{c 0} / p_{h 0}=\left|q_{h}\right| T_{c} /\left|q_{c}\right| T_{h}$. This normalization has been chosen to minimize the number of dimensionless parameters to be considered in the subsequent discussions.

Note also that $\tau$ is not necessarily smaller than 1, as long as the strict inequality $c_{t c}<c_{t h}$ is maintained, otherwise there can be no solitons. In normalized variables, the condition $c_{t c}<V<c_{t h}$ becomes $\sqrt{\tau}<M<1 / \sqrt{\mu}$, so that $\tau$ must be strictly less than $1 / \mu$.

Consequently, we find that

$$
\begin{aligned}
\frac{n_{c}}{n_{c 0}} & =\frac{1}{2 \sqrt{\tau}}\left[\sqrt{(M+\sqrt{\tau})^{2}-2 \varphi}-\sqrt{(M-\sqrt{\tau})^{2}-2 \varphi}\right] \\
\frac{n_{h}}{n_{h 0}} & =\frac{1}{2}\left[\sqrt{(M \sqrt{\mu}+1)^{2}+2 \varphi}+\sqrt{(M \sqrt{\mu}-1)^{2}+2 \varphi}\right]
\end{aligned}
$$

and the constituent densities are coupled through Poisson's equation,

$$
\frac{d^{2} \varphi}{d x^{2}}+\frac{n_{c}}{n_{c 0}}-\frac{n_{h}}{n_{h 0}}=0 .
$$


Here $x$ is a dimensionless coordinate, scaled by $\sqrt{\varepsilon_{0} T_{h} / n_{h 0} q_{h}^{2}}$, the subsonic species' Debye length. After multiplying (2.4) by $d \varphi / d x$ and integrating, one gets

$$
\frac{1}{2}\left(\frac{d \varphi}{d x}\right)^{2}+S(\varphi)=0 .
$$

This is formally equivalent to the energy integral for a particle with unit mass in a conservative force field, with $\varphi$ in the role of the particle coordinate and $x$ in the role of time. The potential energy $S(\varphi)$ is called the Sagdeev potential or pseudopotential (Sagdeev 1966; Sagdeev and Galeev 1969), and is obtained as

$$
\begin{aligned}
S(\varphi)= & M^{2}(1+\mu)+\frac{1}{6 \sqrt{\tau}}\left\{\left[(M-\sqrt{\tau})^{2}-2 \varphi\right]^{3 / 2}-\left[(M+\sqrt{\tau})^{2}-2 \varphi\right]^{3 / 2}\right\} \\
& +\frac{1+\tau}{3}-\frac{1}{6}\left\{\left[(M \sqrt{\mu}+1)^{2}+2 \varphi\right]^{3 / 2}+\left[(M \sqrt{\mu}-1)^{2}+2 \varphi\right]^{3 / 2}\right\} .
\end{aligned}
$$

The discussion can thus be given with this classical mechanical analogy in mind.

\section{Discussion}

It is seen that $S(0)=0$ and $(d S / d \varphi)(0)=0$, and in order to have the possibility of solitary structures, we need the proper convexity at the origin, namely that

$$
\frac{d^{2} S}{d \varphi^{2}}(0)=\frac{1}{M^{2}-\tau}-\frac{1}{1-M^{2} \mu}<0 .
$$

The origin is now a local maximum for $S(\varphi)$, viz., $S(\varphi)<0$ for $\varphi \neq 0$ but in the immediate vicinity of $\varphi=0$. From (3.1) it follows that one requires

$$
M^{2}>M_{s}^{2}:=\frac{1+\tau}{1+\mu} .
$$

This lower bound for $M$, and thus also for $V$, corresponds to the true ion-acoustic velocity in the plasma under consideration. As long as $\tau<1 / \mu$ holds, one can prove that $\sqrt{\tau}<M_{s}<1 / \sqrt{\mu}$ and there is indeed a soliton existence range possible for $M_{s}<M$, although the detailed analysis, discussed below, indicates that usually upper limits on $M$ occur before $1 / \sqrt{\mu}$ is reached and the model breaks down.

An upper limit for $M$ can be found from the fluid-dynamical argument that the description breaks down when, for this simple plasma composition, the supersonic ions reach their sonic point (McKenzie 2002; McKenzie and Doyle 2003; Verheest et al. 2004). It can be shown (Verheest et al. 2008) that this corresponds precisely to the limiting value of $\varphi, \varphi_{\ell}$, for which the adiabatic supersonic ion density [see expression (2.2)] ceases to be real,

$$
\varphi_{\ell}=-\frac{q_{h} \phi_{\ell}}{T_{h}}=\frac{1}{2}(M-\sqrt{\tau})^{2} .
$$

Note that the product $q_{h} \phi_{\ell}$ is negative, and since $q_{c}$ and $q_{h}$ have opposite signs, this means that $\phi_{\ell}$ has the sign of $q_{c}$. For positive/negative supersonic ions the solitons are positive/negative.

In the sonic point the supersonic ion density becomes $\left(n_{c} / n_{c 0}\right)_{\ell}=\sqrt{M / \sqrt{\tau}}$, which is finite as long as $\tau \neq 0$. In the limit $\tau=0$, this corresponds to infinite supersonic ion compression. There is also a limitation on the other side, occurring 


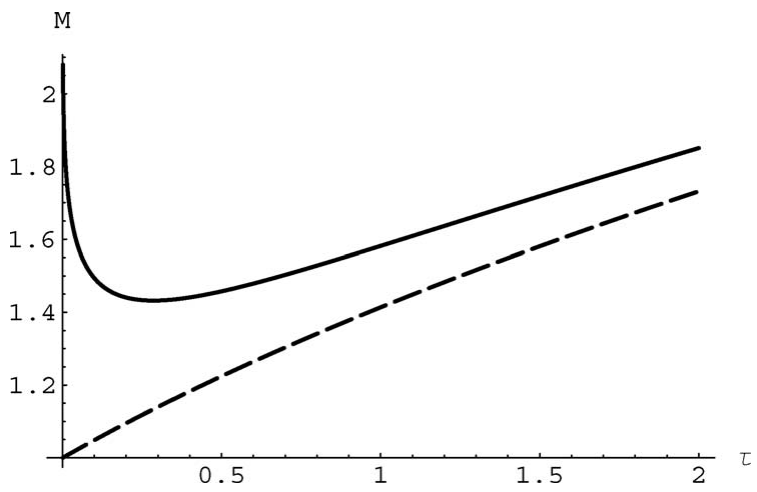

Figure 1. Existence domains for solitons. The dashed lower curve gives $M_{s}$ and the full upper one $M_{\ell}$, as functions of $\tau$, for the typical electron-proton mass ratio $\mu=1 / 1836$.

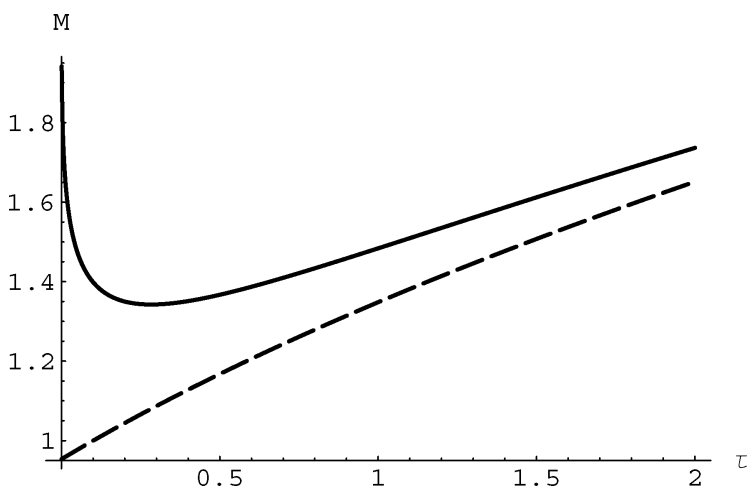

Figure 2. Existence domains for solitons, as in Fig. 1, but for $\mu=1 / 10$.

at the subsonic species' sonic point, but since there are no solitons of that kind, we need not pursue this further.

Hence, if one wants to find a soliton solution, $S(\varphi)$ must have a root, $\varphi_{r}$, before the supersonic ions reach their sonic point at $\varphi_{\ell}$. For $|\varphi|<\left|\varphi_{r}\right|$, the pseudopotential $S(\varphi)$ stays negative. Towards the end of this Section we prove analytically that increasing $M$ means that the soliton amplitude becomes larger and thus $\varphi_{r}$ is pushed farther out, towards $\varphi_{\ell}$. Consequently, the upper limit on $M$, which we call $M_{\ell}$, is computed from $S\left(\varphi_{\ell}\right)=0$.

Both limitations, $M_{s}$ and $M_{\ell}$, can be plotted in various ways to obtain the soliton existence domain in parameter space. Since there are only two compositional parameters to be considered, $\tau$ and $\mu$, we will choose a given $\mu$ and plot $M_{s}$ and $M_{\ell}$ as functions of $\tau$. Examples are given in Figs 1-3, for some typical $\mu$. These figures have to be understood as follows: one picks a given $\tau$, and then any value of $M$ between the lower and the upper curve yields a Sagdeev pseudopotential $S(\varphi)$ having a soliton. Curves can be extended in $\tau$ until $1 / \mu$ is reached, corresponding to equal thermal velocities, but for graphical clarity the graphs have been limited to $\tau=2$ or $\tau=1$, the latter for the case that $\mu=1$.

Figure 1 is focussing on the typical hydrogen plasma, with $\mu=1 / 1836$. In this case, since $q_{c}=-q_{h}=+e,(3.3)$ indicates that in the original, physical notations 


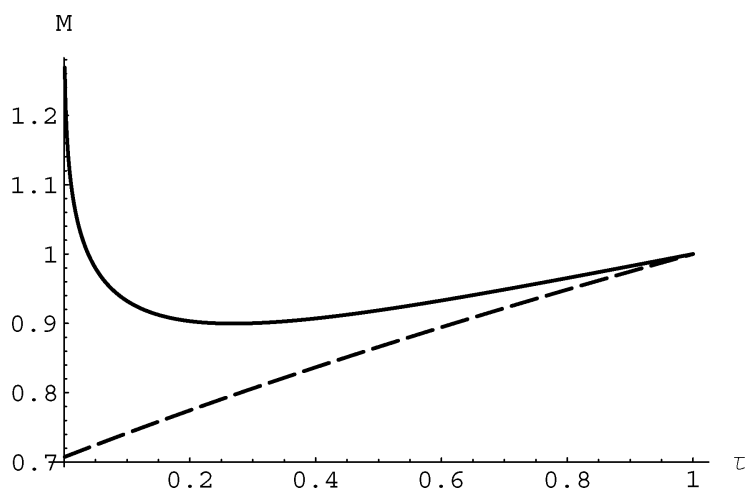

Figure 3. Existence domains for solitons, as in Fig. 1, but for the typical electron-positron mass ratio $\mu=1$.

$\phi_{\ell}>0$, and the ion-acoustic solitons have positive polarity, as is well known from earlier studies (Sagdeev 1966). Were one to redo (as we have done) the same exercise for $\mu=0$ instead of $\mu=1 / 1836$, the graphs would look indistinguishable to the naked eye, differences being of the order of a fraction of a percent or less. Hence, whether one uses the correct electron-proton mass ratio or completely neglects electron inertia, by putting $\mu=0$, does not seem to matter much for adiabatic pressure changes in a two-component plasma. Similar conclusions apply to dusty plasmas in which all electrons have been accreted onto the (negative) dust grains, in the presence of protons or positive ions, once the normalization is adapted to much smaller mass density ratios.

Other cases for $\mu$ are seen in Fig. 2 , for $\mu=1 / 10$, and in Fig. 3 for $\mu=1$. The mass ratio $\mu=1 / 10$ is one of the values of $\mu$ used by Mamun and Shukla (2002) in their study of cometary dusty plasmas. They consider a plasma of negatively and positively charged grains, assuming a complete depletion of background electrons and ions. In this model, heavier dust grains are negatively charged because of preferential capture and lower secondary emission of electrons, whereas lighter grains are positively charged due to photo emission in the presence of ultraviolet photons or by secondary emission of electrons from the surface (Chow et al. 1993).

Increasing $\mu$ to 1 , the electron-positron mass ratio, shows that at equal temperatures $(\tau=1)$ there can be no acoustic modes: the two thermal speeds are equal, and so the model breaks down as the sound speed no longer lies between the two thermal speeds (Verheest 2005; Dubinov et al. 2006; Verheest 2006). When the electrons are hotter than the positrons, the solitons have positive polarity. Upon reversal of this condition one obtains negative solitons. Of course, there is a long standing debate in the literature as to what physical reasons could cause a temperature imbalance between two such symmetric species (Verheest et al. 1996). This discussion also applies to recent fullerene plasma experiments (Oohara and Hatakeyama 2003).

Examples of Sagdeev pseudopotentials are shown in Figs 4-6, and in drawing the graphs we have assumed that $q_{c}$ is positive, to fix the ideas. For $q_{c}<0$, one just flips the $\varphi$ axis around.

In Fig. 4 it is seen that increasing $\mu$ increases the soliton amplitude, at fixed $M$ and $\tau$. This can also be proved analytically and requires that curves for larger $\mu$ have to lie below those for smaller $\mu$. This is equivalent to saying that 


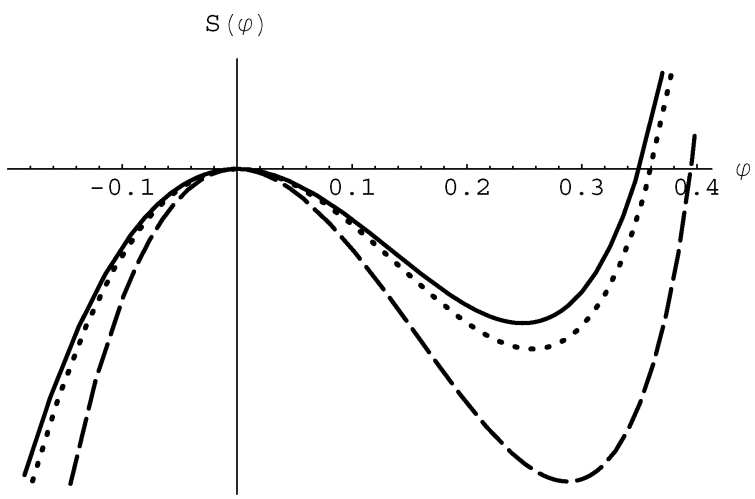

Figure 4. Sagdeev pseudopotentials for $M=1.34, \tau=0.2$ and $\mu=1 / 1836$ (full curve), $\mu=1 / 50$ (dotted curve) and $\mu=1 / 10$ (dashed curve), respectively. The graphs have not been drawn up to the sonic point limits, for graphical clarity, except for the dashed curve on the positive side.

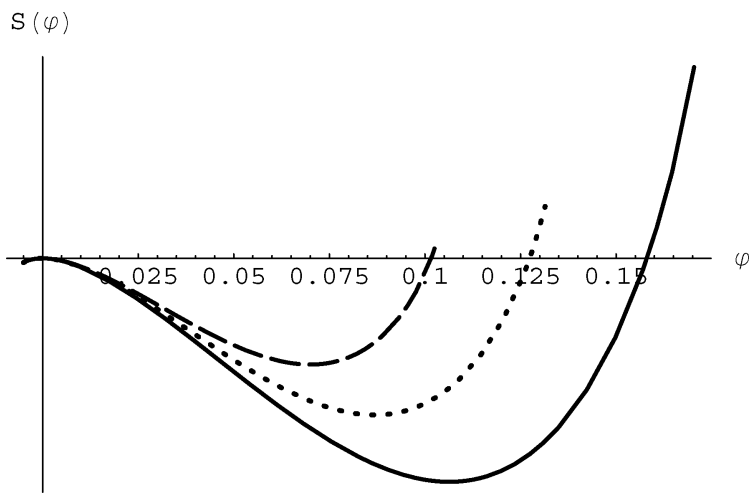

Figure 5. Sagdeev pseudopotentials for $M=0.9$ and $\mu=1$, and $\tau=0.1$ (full curve), $\tau=0.15$ (dotted curve) and $\tau=0.2$ (dashed curve), respectively. The curves have been drawn for the full existence range, from the electron sonic point on the negative side to the positron sonic point on the positive side.

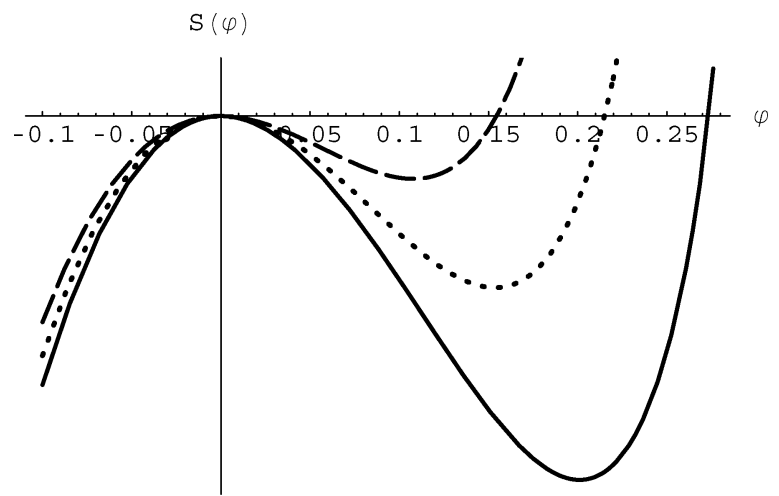

Figure 6. Sagdeev pseudopotentials for $\mu=1 / 1836, \tau=0.5$ and $M=1.45$ (full curve), $M=1.40$ (dotted curve) and $M=1.35$ (dashed curve), respectively. The curves have been drawn for negative $\varphi$ only up to -0.1 and limited for positive $S(\varphi)$ for graphical clarity, except for the full curve which ends in the proton sonic point on the positive side. 
$S(\varphi ; M, \mu, \tau)>S(\varphi ; M, \mu+\delta \mu, \tau)$ for all $\delta \mu>0$ and for all $\varphi \neq 0$ (restricted to the range where all square roots are real). Consequently, we have to prove that

$$
\lim _{\delta \mu \rightarrow 0} \frac{S(\varphi ; M, \mu+\delta \mu, \tau)-S(\varphi ; M, \mu, \tau)}{\delta \mu}=\frac{\partial S(\varphi ; M, \mu, \tau)}{\partial \mu}<0 .
$$

We first note that $\mu$ occurs only in the contribution of the subsonic species to the Sagdeev pseudopotential. By inverting (2.3) and rationalizing the denominator in $n_{h 0} / n_{h}$ we get

$$
\sqrt{(M \sqrt{\mu}+1)^{2}+2 \varphi}-\sqrt{(M \sqrt{\mu}-1)^{2}+2 \varphi}=\frac{2 M \sqrt{\mu} n_{h 0}}{n_{h}} .
$$

When computing $\partial S(\varphi ; M, \mu, \tau) / \partial \mu$ one can replace the large square roots containing $\varphi$ in terms of powers of $n_{h}$ and show that indeed

$$
\frac{\partial S(\varphi ; M, \mu, \tau)}{\partial \mu}=-M^{2} \frac{\left(n_{h}-n_{h 0}\right)^{2}}{2 n_{h} n_{h 0}}<0 .
$$

The strict inequality is valid outside the undisturbed conditions $\left(n_{h} \neq n_{h 0}\right)$. As $M$ is the soliton speed relative to a fixed normalizing speed, $V_{i a}$, it follows that fixed $M$ represents a fixed soliton speed measured in, say $\mathrm{m} \mathrm{s}^{-1}$. The observed trend of soliton amplitudes increasing with $\mu$ appears to be related to the corresponding decrease in $M_{s}$, which results in increasing $M-M_{s}$, e.g. $M-M_{s}=0.245$ for $\mu=1 / 1836$, rising to $M-M_{s}=0.296$ for $\mu=1 / 10$.

It is also worthwhile to consider $M / M_{s}$, as that allows for the fact that the true sound speed changes with changes in $\tau$ and/or $\mu$. Thus $M / M_{s}$, the soliton speed $V$ measured in units of the true sound speed, is actually the true Mach number. Seen from this point of view $M / M_{s}>1$ corresponds to the usual requirement for soliton existence, i.e. the structure is super-acoustic. As $\mu$ is increased, other compositional parameters remaining equal, (3.2) shows that $M_{s}$ is decreased, so $M / M_{s}$ is increased, as is the soliton amplitude.

As can be seen from (2.5), the depth of the pseudopotential well represents the steepest slope of the soliton potential profile. Figure 4 also shows that the steepness of the soliton profile increases with $\mu$, i.e. the width of the profile decreases. Moreover, for all mass ratios between $\mu=0$ and $\mu=1 / 50$, the Sagdeev pseudopotentials lie very close to each other, indicating that small changes in $\mu$ do not matter much. This is supported by a comparison of the existence domains shown in Figs $1(\mu=1 / 1836)$ and $2(\mu=1 / 10)$. It is only when $\mu$ becomes of order unity that significant changes are observed.

On the other hand, it is seen in Fig. 5 that increasing $\tau$ decreases both the soliton amplitude and the soliton profile steepness, at fixed $M$ and $\mu$. An analytic proof, analogous to the one used to obtain (3.6), but for changes in $\tau$, can also be given here. Inversion of (2.2) gives

$$
\sqrt{(M+\sqrt{\tau})^{2}-2 \varphi}+\sqrt{(M-\sqrt{\tau})^{2}-2 \varphi}=\frac{2 M n_{c 0}}{n_{c}}
$$

which, combined with (2.2), allows us to write

$$
\sqrt{(M \pm \sqrt{\tau})^{2}-2 \varphi}=\frac{M n_{c 0}}{n_{c}} \pm \frac{n_{c} \sqrt{\tau}}{n_{c 0}} .
$$


Since $\tau$ occurs only in the contribution of the supersonic ions to (2.6), one readily obtains

$$
\frac{\partial S(\varphi ; M, \mu, \tau)}{\partial \tau}=\frac{\left(n_{c}-n_{c 0}\right)^{2}\left(n_{c}+2 n_{c 0}\right)}{6 n_{c 0}^{3}}>0 .
$$

Now curves for larger $\tau$ lie above those for smaller $\tau$, as is clearly seen in Fig. 5 . Again, this is probably related to the corresponding increase in $M_{s}$ with $\tau$, which gives $M-M_{s}=0.158$ for $\tau=0.1$, decreasing to $M-M_{s}=0.125$ for $\tau=0.2$. Here, as $\tau$ is increased, (3.2) shows that $M_{s}$ is increased, so $M / M_{s}$ is decreased, as is the soliton amplitude, with a concomitant increase in width of the soliton profile.

Finally, one can prove that curves for larger $M$ lie below those for smaller $M$, all compositional parameters being equal. With the help of (2.3), (3.5) and (3.8) we now find that

$$
\frac{\partial S(\varphi ; M, \mu, \tau)}{\partial M}=-M\left[\frac{\left(n_{c}-n_{c 0}\right)^{2}}{n_{c} n_{c 0}}+\mu \frac{\left(n_{h}-n_{h 0}\right)^{2}}{n_{h} n_{h 0}}\right]<0 .
$$

This trend is illustrated in Fig. 6. As $M$ is increased, other compositional parameters remaining equal, it is trivial to remark that $M / M_{s}$ is increased, as are, also, the soliton amplitude and the soliton profile steepness.

\section{Conclusions}

We have investigated, through a Sagdeev pseudopotential analysis, large amplitude ion-acoustic solitons in a plasma with two adiabatic constituents, with the full inclusion of inertial and pressure effects for both. Emphasis has been placed on the determination of the soliton existence domains in compositional parameter space, allowing correct Sagdeev pseudopotential graphs to be easily generated, and offering insight into why limitations occur. Soliton velocities are bounded from below by the true acoustic velocity in the plasma model, and from above by the breakdown of the description when the supersonic ions reach their sonic point.

In the normalization used in this paper, the strongest solitons occur for $\tau$ small, in other words, a higher temperature of the supersonic (cooler) species adversely affects soliton amplitudes. On the other hand, the effect of $\mu$ is much less pronounced, except when $\mu$ tends to 1 . For standard electron-proton plasmas, electron inertia does not have much influence on the physics. At the same time, since for reasonable values of $\tau$ we have that $M$ is of order $1 \sim 2$, over the whole $\mu$ range, soliton amplitudes will be of the same order or less; they might be of significant amplitude, but cannot be excessively large.

Further applications of this study include dusty plasmas in which all electrons have been accreted onto the dust grains, although the conclusions here are qualitatively very similar to what obtains for hydrogen plasmas, once the normalization is adapted to much larger mass-per-charge ratios for the heavy dust grains. Intermediate values for $\mu$ can be obtained in models where there is a mixture of heavier, negatively charged dust in the presence of lighter, positively charged grains, when there is a near complete depletions of the original plasma particles, as may be useful for cometary studies.

\section{Acknowledgement}

M. A. H. thanks the National Research Foundation for research grants. Any opinion, findings and conclusions or recommendations expressed in this material are 
those of the authors and therefore the NRF does not accept any liability in regard thereto.

\section{References}

Bliokh, P., Sinitsin, V. and Yaroshenko, V. 1995 Dusty and Self-Gravitational Plasmas in Space. Dordrecht, The Netherlands: Kluwer Academic.

Chow, V. W., Mendis, D. A. and Rosenberg, M. 1993 Role of grain size and particle velocity distribution in secondary electron emission in space plasmas. J. Geophys. Res. 98, 1906519076.

Dubinov, A. E., Dubinova, I. D. and Gordienko, V. A. 2006 Solitary electrostatic waves are possible in unmagnetized symmetric pair plasmas. Phys. Plasmas 13, 082111.

Ghosh, S. S., Ghosh, K. K. and Sekar Iyengar, A. N. 1996 Large Mach number ion acoustic rarefactive solitary waves for a two electron temperature warm ion plasma. Phys. Plasmas 3, 3939-3946.

Mamun, A. A. 2008 Effect of adiabaticity of electrons and ions on dust-ion-acoustic solitary waves. Phys. Lett. A 372, 1490-1493.

Mamun, A. A., Jahan, N. and Shukla, P. K. 2009 DIA and DA solitary waves in adiabatic dusty plasmas. J. Plasma Phys. 75, 413-431.

Mamun, A. A. and Shukla, P. K. 2002 Solitary potentials in cometary dusty plasmas. Geophys. Res. Lett. 29, 1870, doi:10.1029/2002GL015219.

McKenzie, J. F. 2002 The ion-acoustic soliton: a gas-dynamic point of view. Phys. Plasmas 9, 800-805.

McKenzie, J. F. and Doyle, T. B. 2003 A unified view of acoustic-electrostatic solitons in complex plasmas. New J. Phys. 5, 26.

Oohara, W. and Hatakeyama, R. 2003 Pair-ion plasma generation using fullerenes. Phys. Rev. Lett. 91, 205005.

Pillay, S. R. and Bharuthram, R. 1992 Large amplitude solitons in a multi-species electronpositron plasma. Astrophys. Space Sci. 198, 85-93.

Rao, N. N., Shukla, P. K. and Yu, M. Y. 1990 Dust-acoustic waves in dusty plasmas. Planet. Space Sci. 38, 543-546.

Sagdeev, R. Z. 1966 Cooperative phenomena and shock waves in collisionless plasmas. In: Reviews of Plasma Physics, Vol. 4 (ed. M. A. Leontovich). New York: Consultants Bureau, pp. 23-91.

Sagdeev, R. Z. and Galeev, A. A. 1969 Nonlinear Plasma Theory. New York: W. A. Benjamin.

Shukla, P. K. and Mamun, A. A. 2002 Introduction to Dusty Plasma Physics. London: IOP Press.

Srinivas, J., Popel, S. I. and Shukla, P. K. 1996 Electrostatic solitons in an electron-positron plasma with two distinct groups of positrons. J. Plasma Phys. 55, 209-217.

Tanjia, F. and Mamun, A. A. 2009 Adiabatic effects of electrons and ions on electro-acoustic solitary waves in an adiabatic dusty plasma. J. Plasma Phys. 75, 99-110.

Verheest, F. 2000 Waves in Dusty Space Plasmas. Dordrecht, The Netherlands: Kluwer.

Verheest, F. 2005 On the nonexistence of large amplitude stationary solitary waves in symmetric unmagnetized pair plasmas. Nonl. Proc. Geophys. 12, 569-574.

Verheest, F. 2006 Existence of bulk acoustic modes in pair plasmas. Phys. Plasmas 13, 082301.

Verheest, F., Hellberg, M. A., Gray, G. J. and Mace, R. L. 1996 Electrostatic solitons in multispecies electron-positron plasmas. Astrophys. Space Sci. 239, 125-139.

Verheest, F., Cattaert, T., Lakhina, G. S. and Singh, S. V. 2004 Gas-dynamic description of electrostatic solitons. J. Plasma Phys. 70, 237-250.

Verheest, F., Hellberg, M. A. and Lakhina, G. S. 2007 Necessary conditions for the generation of acoustic solitons in magnetospheric and space plasmas with hot ions. Astrophys. Space Sci. Trans. 3, 15-20.

Verheest, F., Hellberg, M. A. and Kourakis, I. 2008 Acoustic solitary waves in dusty and/or multi-ion plasmas with cold, adiabatic and hot constituents. Phys. Plasmas 15, 112309. 